\title{
A novel TAB2 nonsense mutation (p.S149X) causing autosomal dominant congenital heart defects: a case report of a Chinese family
}

Jia Chen ${ }^{1}$, Huizhen Yuan ${ }^{1}$, Kang Xie ${ }^{1}$, Xinrong Wang ${ }^{1}$, Linglong Tan ${ }^{1}$, Yongyi Zou', Yan Yang ${ }^{1}$, Lu Pan ${ }^{1}$, Junfang Xiao ${ }^{1}$, Ge Chen ${ }^{2}$ and Yanqiu Liu ${ }^{1 *}$ (D)

\begin{abstract}
Background: TAB2 is an activator of MAP 3 K7/TAK1, which is required for the IL-1 induced signal pathway. Microdeletions encompassing TAB2 have been detected in various patients with congenital heart defects (CHD), indicating that haploinsufficiency of TAB2 causes CHD. To date, seven variants within TAB2 were reported associated with $\mathrm{CHD}$, only two of them are nonsense mutations.

Case presentation: Here we describe a three-generation Chinese family that included five CHD patients with heart valvular defects, such as mitral or tricuspid valves prolapse or regurgitation, and aortic valve stenosis or regurgitation. Our proband was a pregnant woman presenting with mitral, tricuspid, and aortic defects; her first child experienced sudden cardiac death at the age of 2 years. Whole-exome sequencing of the proband revealed a novel nonsense variant in TAB2 (c.C446G, p.S149X), which results in the elimination of the majority of C-terminal amino acids of TAB2, including the critical TAK1-binding domain. The variant was identified in five affected patients but not in the eight unaffected family members using Sanger sequencing and was classified as "pathogenic" according to the latest recommendation on sequence variants laid out by the American College of Medical Genetics and Genomics and the Association for Molecular Pathology.

Conclusion: We described a family with CHD caused by a novel TAB2 nonsense mutation. Our study broadens the mutation spectrum of TAB2; to the best of our knowledge, this is the first report of a pathogenic mutation within TAB2 in a Chinese population.
\end{abstract}

Keywords: Congenital heart defects, Valvular anomalies, TAB2, Whole-exome sequencing

\section{Background}

TAB2 is a gene located on chromosome 6q25.1 [OMIM "605101] and encodes the TGF- $\beta$-activated kinase 1/ MAP $3 \mathrm{~K} 7$ binding protein 2 (TAB2). As an adapter protein linking TGF- $\beta$-activated kinase 1 (TAK1/MAP 3 K7) and TNF receptor-associated factor 6 (TRAF6), TAB2 plays an essential role in the activation of JNK/NF- $\mathrm{KB}$ signaling induced by IL-1 [1]. Haploinsufficiency of $T A B 2$ has been linked to congenital heart defects (CHD)

\footnotetext{
* Correspondence: lyq0914@126.com

${ }^{1}$ Prenatal Diagnosis Center, Jiangxi Provincial Maternal and Child Health Hospital, Nanchang 330006, Jiangxi, China

Full list of author information is available at the end of the article
}

via mapping of the smallest overlapping region of various 6q25.1 microdeletions in different patients with CHD [2]. Further investigations have shown that TAB2 is expressed in embryonic cardiac tissues of both humans and zebrafish. Knocking down TAB2 in zebrafish embryos resulted in delayed epiboly progression, convergent extension defects during gastrulation (at approximately $12 \mathrm{~h}$ postfertilization), and severe heart failure 36-48 $\mathrm{h}$ postfertilization [2]. These findings indicate that TAB2 dysfunction causes CHD. Additionally, TAB2 mutations have also been found in patients with frontometaphyseal dysplasia (FMD), with apparently different phenotypes from CHD [3, 4]. 
According to the latest Human Gene Mutation Database (HGMD; http://www.hgmd.cf.ac.uk/ac/index.php), 21 deletions encompassing TAB2 and seven diseasecausing variants within the gene have been associated with CHD; only two of them are nonsense mutations. Here we examined a three-generation Chinese family including eight patients with CHD (Fig. 1a; Table 1). Heart valvular defects were detected in most affected patients by standard echocardiography. A novel heterozygous variant (c.C446G) in exon5 of TAB2 was detected in the proband by Whole-Exome Sequencing (WES) and identified in all the surviving affected family members using Sanger sequencing (Fig. 1b; Table 1). This nonsense variant creates a premature stop codon at the 149th residue of TAB2 (p.S149X) and removes the majority of amino acids from the protein, including the TAK1-binding domain (TAK1 BD) (Fig. 2) [1]. This variant is classified as "pathogenic" as per the latest recommendation on sequence variants interpretation laid out by the American College of Medical Genetics and Genomics (ACMG) and the Association for Molecular Pathology (AMP) [5]. Our study expands the variant diversity of $T A B 2$ and provide more clinical symptoms of CHD patients caused by a TAB2 mutation in a Chinese population.

\section{Case presentation}

\section{Clinical information}

The study was approved by the institutional review board of Jiangxi maternal and child health hospital, Nanchang, China. All the enrolled subjects provided written informed consent. A family from the Jiangxi province, China, comprising 25 family members (11 males, 12 females, and 2 fetuses) across three generations (Fig. 1a) were included in the study. Our proband (II:5) was a 31-year-old female who was 15 weeks pregnant at her first visit to our hospital. She was slightly tachypneic and reported occasional fatigue since the age of 25 years old. She performed a transthoracic color echocardiogram examination at 30 years old and revealed mild aortic valve stenosis accompanied with mild mitral, tricuspid, and aortic regurgitation. She denied any surgical or pharmaceutical interventions. Her husband was 32 years old (II:6) and declared no cardiac symptoms or family history of heart diseases. Their first child (III:7) was a CHD girl with left and right ventricles dilatation. The girl then experienced sudden cardiac death (SCD) at 2 years of age. The second child (III:8) was detected with no heartbeat at 9 weeks of pregnancy using a Doppler fetal monitor, and subsequently, the pregnancy was
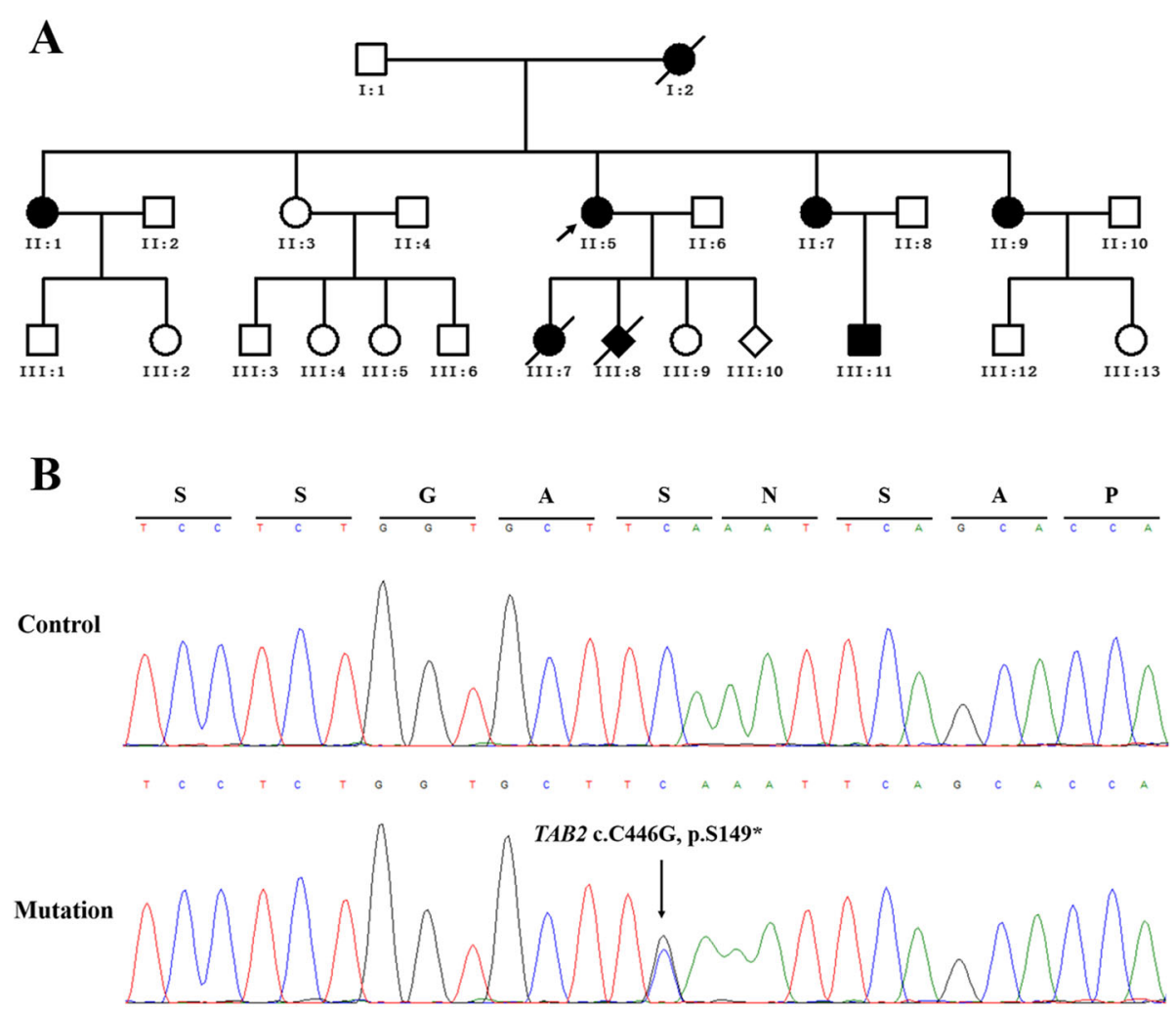

Fig. 1 Pedigree of the CHD family and sequence results of the TAB2 mutation. a All sampled subjects in the pedigree are identified by Roman numerals below the symbol. Arabic numbers denote each individual in a generation. Open symbols, unaffected; filled symbols, affected; symbols with a diagonal line, deceased subjects; squares, male; circles, female; diamond, fetus with unknown sex; arrow, the proband. b Sequence chromatogram indicates a C-to-G transition of nucleotide 466 
Table 1 Summary of the CHD family

\begin{tabular}{|c|c|c|c|c|c|}
\hline \multirow{2}{*}{$\begin{array}{l}\text { CHD Family } \\
\text { members }\end{array}$} & \multirow[t]{2}{*}{$\mathrm{CHD}$} & \multirow{2}{*}{$\begin{array}{l}\text { Age at } \\
\text { diagnosis }\end{array}$} & \multirow[t]{2}{*}{ Heart defects according to transthoracic echocardiogram } & \multicolumn{2}{|l|}{ TAB2 } \\
\hline & & & & DNA & Protein \\
\hline$\|: 1$ & Yes & $39 y$ & $\begin{array}{l}\text { atrial septal aneurysm, left coronary artery dilation, } \\
\text { mild aortic regurgitation }\end{array}$ & c.C446G & p.S149X \\
\hline$\|: 3$ & No & $36 y$ & - & - & - \\
\hline$\|: 5$ & Yes & $31 y$ & $\begin{array}{l}\text { mild mitral and tricuspid regurgitation, mild aortic } \\
\text { valve stenosis with aortic regurgitation }\end{array}$ & c.C446G & p.S149X \\
\hline$\|: 6$ & No & $32 y$ & - & - & - \\
\hline$\|: 7$ & Yes & $30 y$ & $\begin{array}{l}\text { atrial septal aneurysm, mild mitral valves prolapse with } \\
\text { mitral regurgitation, mild pulmonic regurgitation }\end{array}$ & C.C446G & p.S149X \\
\hline$\|: 8$ & No & $31 y$ & - & - & - \\
\hline$\|: 9$ & Yes & $29 y$ & atrial septal aneurysm, left atrial and ventricular dilatation & c.C446G & p.S149X \\
\hline |ll:3 & No & $12 y$ & - & - & - \\
\hline$\| 11: 4$ & No & $11 y$ & - & - & - \\
\hline |ll:5 & No & $3 y$ & - & - & - \\
\hline \|ll:9 & No & $2 y$ & - & - & - \\
\hline$\|1\|: 10$ & High risk & 19w pregnancy & - & c.C446G & p.S149X \\
\hline |II:11 & Yes & $3 y$ & $\begin{array}{l}\text { mild left ventricular and right atrial dilation, mild } \\
\text { mitral valves prolapse with mitral regurgitation }\end{array}$ & c.C446G & p.S149X \\
\hline |ll:13 & No & $1 y$ & - & - & - \\
\hline
\end{tabular}

CHD Congenital heart defect, $y$ Years old, $w$ Weeks; "-", no defect or mutation detected

terminated. The third child (III:9) was a 2-year-old girl with no symptoms of cardiac disorders. She was not diagnosed with any heart abnormalities using echocardiography.

The proband's other family members were interviewed, and cardiac symptoms, such as tachypnea, shortness of breath, and fatigue, were reported. Four females and one male were identified with clinical symptoms (Fig. 1a). The proband's mother (I:2) died in her late fifties due to sudden cardiac arrhythmia. Evident atrial septal aneurysms were detected in the proband's sisters (II:1, II:7, and II:9) using echocardiography. Left coronary artery dilation was screened in the patient II:1 by echocardiography and then identified using coronary arteriography. Other cardiac anomalies, such as mild mitral valves prolapse with mitral and pulmonic regurgitation, and left atrial and ventricular dilatation, were found in II:7 and II:9, respectively. III:11 was a 3-year-old boy who was

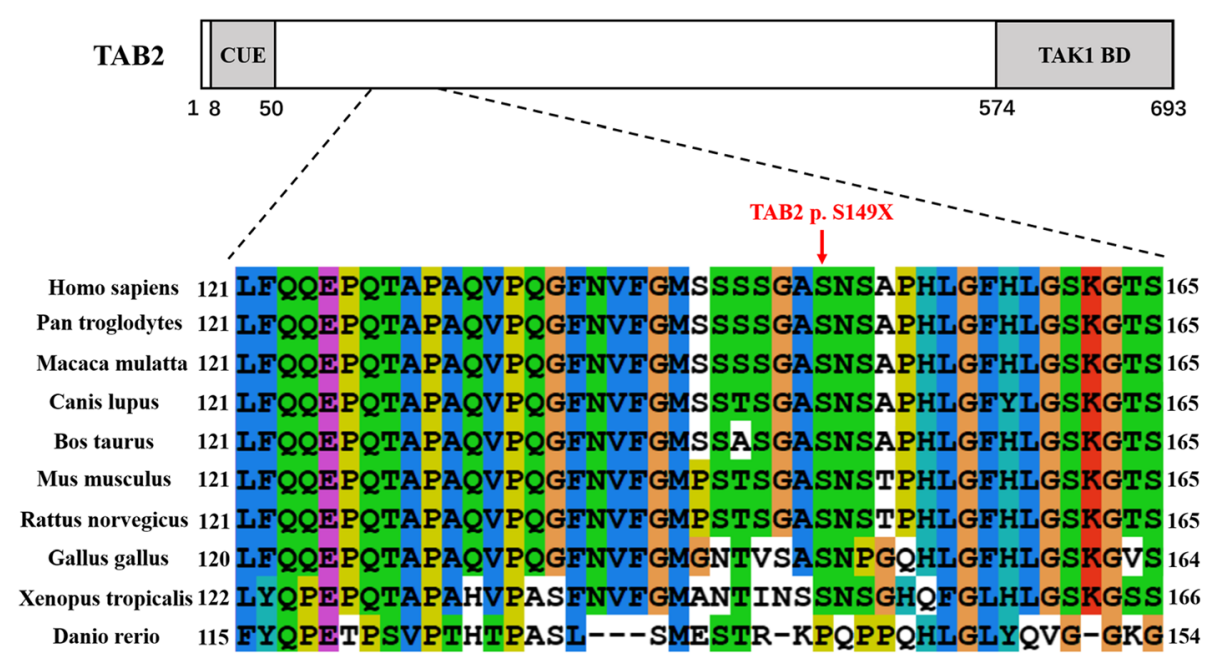

Fig. 2 Analysis of protein domains and the mutation of TAB2. Schematic representation of TAB2 protein domains indicate an N-terminal coupling of ubiquitin conjugation to endoplasmic reticulum (CUE) domain and a C-terminal TAK1 binding domain (TAK1 BD). The affected amino acid S149, indicated by red arrow, is highly conserved in different species 
diagnosed with mild left ventricular and right atrial dilation as well as mild mitral valves prolapse with mitral regurgitation using echocardiography. Transabdominal fetal echocardiography was performed on the proband's fetus (III:10) at 15 weeks of pregnancy, and no cardiac abnormality was detected. The heart defects in the individuals enrolled in this study are summarized in Table 1.

\section{Mutation detection}

An autosomal dominant inheritance pattern was suggested on the basis of vertical transmission of CHD in the family. Peripheral blood was obtained from 13 family members (II:1, II:3, II:5, II:6, II:7, II:8, II:9, III:3, III:4, III: 5, III:9, III:11, and III:13). Genomic DNA was extracted from the peripheral blood lymphocytes using the QIAamp DNA blood mini kit (Qiagen).

To determine the causative mutation in the family, WES of the proband (II:5) was performed as previously described with minor modifications [6]. Two micrograms of the genomic DNA from the proband II:5 was used for human whole-exome analysis with paired-endsequencing at $100 \times$ resolution. Libraries were constructed using the SureSelect Human All ExonV7 kit (Agilent Technologies, USA) and sequenced on the Illumina HiSeq 2500 platform (Illumina, San Diego, CA), as per the manufacturer's instructions. The reads were aligned to the human reference genome (University of California Santa Cruz, UCSC hg19) using SOAPaligner. Single-nucleotide polymorphism (SNP) and indel (insertion or deletion) identification was performed using SAMtools and/or the Genome Analysis Toolkit (GATK), and SNPs with a read depth $>4$ and quality $>20$ were used for subsequent analyses. SNPs and indels were annotated using SeattleSeq annotation. Known polymorphisms in the dbSNP (https://www.ncbi.nlm.nih.gov/snp/) (minor allele frequency, >0.01) and 1000 genomes (https:// www.ncbi.nlm.nih.gov/variation/tools/1000genomes/) (genotype frequency, >0.005) databases as well as synonymous single-nucleotide variants and variants not located in exonic or splicing regions were excluded. Pathogenicity of the obtained variants was predicted using Polyphen-2 (http://genetics.bwh.harvard.edu/pph2/index. shtml) [7], SIFT (https://sift.bii.a-star.edu.sg/) [8], and MutationTaster (http://www.mutationtaster.org/) [9].

Approximately $99.71 \%$ of the sequencing reads were mapped to the human genome hg19, with mean $186.76 \times$ sequencing depth. A heterozygous variant in TAB2 (c.C446G, NM_015093.5), which results in a premature stop codon with early termination of protein translation at residue 149 (p.S149X), passed the filtering criteria. Using Sanger sequencing, this variant was detected in all the affected family members (II:1, II:5, II:7, II:9, and III: 11); it was absent in the unaffected individuals (II:3, II:6, II:8, III:3, III:4, III:5, III:9, and III:13) (Fig. 1b). The variant, with no record in 1000 genomes, the Exome Variant Server (EVS; http://evs.gs.washington.edu/EVS/), the Genome Aggregation Database (gnomAD; http://gnomad. broadinstitute.org/), and the HGMD databases, was not detected in our 531 control cohorts using high-resolution melting analysis (SsoFast EvaGreen, Bio-Rad). TAB2 protein sequences (from Danio rerio to Homo sapiens) were obtained from the protein database of the National Center for Biotechnology Information (NCBI, https://www.ncbi. nlm.nih.gov/). Alignments of TAB2 protein family members using cluster 2.0 software [10] revealed that the affected amino acid is evolutionarily conserved (Fig. 2). According to the latest recommendation by ACMG and AMP on sequence variants interpretation, the c.C446G variant in TAB2 would be classified as "pathogenic", having met the requirements of fulfilling one very strong (PVS1), one moderate (PM2) and three supporting (PP1, PP3 and PP4) criteria (Fig. 3) [5].

A prenatal molecular genetic diagnosis for the fetus (III:10) was recommended for evaluating the risk for CHD. With complete informed consent of the proband (II:5) and her husband (II:6), a prenatal diagnosis of the fetus (III:10) was performed at 19 weeks of pregnancy. The amniocytes was obtained by amniocentesis, and fetal genomic DNA (III:10) was extracted using the QIAamp DNA mini kit (Qiagen). A heterozygous TAB2 c. C446G variant was detected, indicating that the fetus had a high risk for CHD.

\section{Discussion and conclusions}

TAB2 is a 693-amino acid protein and plays an important role in the IL-1 signaling pathway and cardiac development. In the present study, a nonsense mutation in $T A B 2$ was identified in five living patients with cardiac defects in a Chinese family. Most patients in the family presented mild clinical symptoms, such as slightly tachypnea and occasional fatigue, except for the proband's first girl child (III:7) who had left and right ventricular dilatation and suffered a SCD at 2 years old. The dilation of left ventricle is a hallmark of dilated cardiomyopathy (DCM) and may contribute to the unexpected death of patient III:7. DCM is defined by the presence of left ventricular dilatation and contractile dysfunction and serves as a leading cause of SCD, especially in childhood $[11,12]$. Patients carrying TAB2 mutations have been associated with DCM. A 7-month-old girl with TAB2 c.1168delT mutation was diagnosed with DCM and conducted a heart transplant surgery at 9 months old, then died at 2.5 years old [13]. DCM was also detected in a 60-year-old brother and a 48-year-old sister who were both carrying a TAB2 c.1398dupT mutation [14]. In addition, arrhythmia, another risk factor of SCD, had been reported in patients with $T A B 2$ mutations $[2,14]$. 


\begin{tabular}{|c|c|c|c|c|c|c|}
\hline & \multicolumn{2}{|c|}{ Benign } & \multicolumn{4}{|c|}{ Pathogenic } \\
\hline & Strong & $\underset{\text { Supporting }}{\longrightarrow}$ & Supporting & Moderate & Strong & Very Strong \\
\hline $\begin{array}{l}\text { Population } \\
\text { Data }\end{array}$ & $\begin{array}{l}\text { MAF frequency is too } \\
\text { high for disorder OR } \\
\text { observation in controls } \\
\text { inconsistent with disease } \\
\text { penetrance }\end{array}$ & & & $\begin{array}{l}\text { Absent in 1000G, EVS } \\
\text { and gnomAD } \\
\text { PM2 }\end{array}$ & $\begin{array}{l}\text { Prevalence in affecteds } \\
\text { statistically increased } \\
\text { over controls } \\
\text { PS4 }\end{array}$ & \\
\hline $\begin{array}{l}\text { Computational } \\
\text { \& Predictive } \\
\text { Data }\end{array}$ & & $\begin{array}{l}\text { Multiple lines of } \\
\text { computational evidence } \\
\text { suggest no impact on } \\
\text { gene /gene product } \\
\text { Missense in gene where } \\
\text { only truncating cause } \\
\text { disease }\end{array}$ & $\begin{array}{l}\text { Multiple lines of } \\
\text { computational } \\
\text { evidence support a } \\
\text { deleterious effect on } \\
\text { the gene /gene product } \\
\text { PP3 }\end{array}$ & $\begin{array}{l}\text { Novel missense change } \\
\text { at an amino acid } \\
\text { residue where a } \\
\text { different pathogenic } \\
\text { missense change has } \\
\text { been seen before } \\
\text { PM5 } \\
\text { Protein length } \\
\text { changing variant } \\
\text { PM4 }\end{array}$ & $\begin{array}{l}\text { Same amino acid } \\
\text { change as an } \\
\text { established pathogenic } \\
\text { variant } \\
\text { PS1 }\end{array}$ & $\begin{array}{l}\text { Predicted null variant } \\
\text { in a gene where LOF is } \\
\text { a known mechanism of } \\
\text { disease } \\
\text { PVS1 }\end{array}$ \\
\hline $\begin{array}{l}\text { Functional } \\
\text { Data }\end{array}$ & $\begin{array}{l}\text { Well-established } \\
\text { functional studies show } \\
\text { no deleterious effect }\end{array}$ & & $\begin{array}{l}\text { Missense in gene with } \\
\text { low rate of benign } \\
\text { missense variants and } \\
\text { path. missense } \\
\text { common } \\
\text { PP2 }\end{array}$ & $\begin{array}{l}\text { Mutational hot spot or } \\
\text { well-studied functional } \\
\text { domain without benign } \\
\text { variation } \\
\text { PM1 }\end{array}$ & $\begin{array}{l}\text { Well-established } \\
\text { functional studies show } \\
\text { a deleterious effect } \\
\text { PS3 }\end{array}$ & \\
\hline $\begin{array}{l}\text { Segregation } \\
\text { Data }\end{array}$ & $\begin{array}{l}\text { Non-segregation with } \\
\text { disease }\end{array}$ & & $\begin{array}{l}\text { Co-segregation with } \\
\text { disease in multiple } \\
\text { affected family } \\
\text { members } \\
\text { PP1 }\end{array}$ & Increased segregation data & & \\
\hline De novo Data & & & & $\begin{array}{l}\text { De novo (without } \\
\text { paternity \& maternity } \\
\text { confirmed) } \\
\text { PM6 }\end{array}$ & $\begin{array}{l}\text { De novo (paternity \& } \\
\text { maternity confirmed) } \\
\text { PS2 }\end{array}$ & \\
\hline Allelic Data & & $\begin{array}{l}\text { Observed in trans with a } \\
\text { dominant variant } \\
\text { Observed in cis with a } \\
\text { pathogenic variant }\end{array}$ & & $\begin{array}{l}\text { For recessive disorders, } \\
\text { detected in trans with a } \\
\text { pathogenic variant } \\
\text { PM3 }\end{array}$ & & \\
\hline $\begin{array}{l}\text { Other } \\
\text { Database }\end{array}$ & & $\begin{array}{l}\text { Reputable database = } \\
\text { benign }\end{array}$ & $\begin{array}{l}\text { Reputable database = } \\
\text { pathogenic } \\
\text { PP5 }\end{array}$ & & & \\
\hline Other Data & & $\begin{array}{l}\text { Found in case with an } \\
\text { alternate cause }\end{array}$ & $\begin{array}{l}\text { Patient's phenotype } \\
\text { highly specific for } \\
\text { gene } \\
\text { PP4 }\end{array}$ & & & \\
\hline
\end{tabular}

Fig. 3 Variant assessment of S149X in TAB2 according to the recommendations of the ACMG and the AMP. Criteria fulfilled by this variant are indicated in yellow. This figure has been adapted from Richards et al. [5]

The heart defects detected using transthoracic echocardiography in the affected members were heterogeneous and mainly involved the heart valves. The proband was diagnosed with mitral, tricuspid, and aortic valve regurgitation. Aortic, pulmonic, and mitral valve dysfunctions were observed in two of her sisters (II:1 and II:7) and one of her nephews (III:11), respectively. In addition, three of the proband's sisters (II:1, II:7, and II:9) had atrial septal aneurysm. Dilation in the coronary artery or heart chambers was detected in patients II:1, II:9, and III:11.

Mutations disrupting TAB2 are associated with heart valvular defects. Several studies have reported that CHD patients with 6q24-q25 microdeletions, containing the $T A B 2$ locus, experience valve anomalies, such as valvular stenosis, mitral or aortic regurgitation, and atrial or ventricular septal defects [2, 14-17]. Similar valvular defects have also been detected in patients with missense, nonsense, and small insertion or deletion mutations within TAB2. A c.622 C>T (p.P208S) and a c.688 C > A (p.Q230K) mutation in TAB2 were identified in a woman with aortic regurgitation and a man with bicuspid aortic valve, respectively [2]. WES of a male child with polyvalvular syndrome revealed a c.1491 T > A nonsense mutation (p.Y497X) in TAB2 [18]. Pulmonary artery aneurysm, moderate mitral regurgitation, and mild tricuspid regurgitation were discovered in a family with a c.1039 C > T nonsense (p.R347X) TAB2 mutation [19]. A c.1398dupT mutation in TAB2 was confirmed in a family with polyvalvular heart disease [14]. Recently, a girl with a de novo TAB2 c.1168delT mutation was diagnosed with dilated cardiomyopathy at 7 months of age; she underwent a heart transplant after 2 months and died at 2.5 years of age [13].

Apart from cardiac defects, TAB2 mutations are associated with connective tissue disorders. The male child with CHD due to the TAB2 p.Y497X mutation also had hypotonia, myopia, soft pale skin, joint hypermobility, and mild facial dysmorphism [18]. Similar clinical features were observed in the family with the TAB2 c.1398dupT mutation [14]. However, no abnormality in the connective tissues was observed in the patients in our study. The family carrying the TAB2 p.R347X nonsense mutation did not show any connective tissue disorders either [19]. These findings suggest clinical heterogeneity of extracardiac tissues in 
patients with TAB2 mutations. In addition, a c.1705 G > A mutation (p.E569K) and a c.1619 A > G mutation (p.Q540R) in TAB2 were detected in patients with FMD, a progressive sclerosing skeletal dysplasia that affects the long bones and the skull. Different from the loss-of-function mutations involved in CHD, these TAB2 mutations cause FMD through a gain-offunction mechanism $[3,4]$.

A detailed understanding of the loss-of-function mutations of TAB2 that cause heart valvular defects remains unclear. The endothelial-to-mesenchymal transition (EndMT) process which endothelial cells migrate from the endocardial layer into the cardiac jelly and acquire mesenchymal characteristics to form the cardiac valves is essential in cardiac valvular development. This process is mainly regulated by the TGF- $\beta$ signaling [20]. Disturbances in the TGF- $\beta$ pathway undermine EndMT [21,22] and cause valvular diseases, such as mitral valve degeneration and myxomatous atrioventricular valve diseases [23, 24]. TAB2 plays an important role in the TGF- $\beta$ pathway by phosphorylating the TAK1/MAP $3 \mathrm{~K} 7$ protein, which then activates NF- $\mathrm{B}$ and MAPK signaling [25]. TAB2 mutations may cause valvular defects by disrupting the EndMT process controlled by the TGF- $\beta$ pathway.

In summary, this study describes a three-generation Chinese family with a history of CHD. In this family, five living patients mainly presented with heart valvular defects. Whole-exome and direct sequencing were used to trace the genetic cause of the disease. We identified the first TAB2 mutation (c.C446G, p.S149X) in a Chinese population. Molecular prenatal diagnosis was performed for the proband's fetus after the mutation was suggested to be pathogenic as per the latest recommendation on sequence variants interpretation laid out by the ACMG. The mutation may disturb cardiac valvular development by impeding the EndMT process regulated by the TGF$\beta$ pathway. Our study broadens the mutation spectrum of the TAB2 gene and implies that TAB2 plays a crucial role in the EndMT process.

\section{Abbreviations \\ ACMG: The American College of Medical Genetics and Genomics; AMP: The Association for Molecular Pathology; CHD: Congenital heart diseases; DCM: Dilated cardiomyopathy; EndMT: Endothelial-to-mesenchymal transition; EVS: Exome variant server database; FMD: Frontometaphyseal dysplasia; GATK: Genome analysis toolkit; gnomAD: Genome aggregation database; HGMD: Human gene mutation database; indel: Insertion or deletion; NCBI: National Center for biotechnology information; SCD: Sudden cardiac death; SNP: Single-nucleotide polymorphism; TAK1 BD: TAK1-binding domain; WES: Whole-exome sequencing}

\section{Acknowledgements}

We thank all of the individuals in the family for their kind agreement to participate in this study.

\section{Authors' contributions}

$Y L$ and $J C$ designed the study. $Y L, J C$, and $Y Z$ drafted the manuscript; JC and $Y Z$ performed the sequencing and the high-resolution melting analysis experiments; HY, LT, LP, and JX collected the clinical information; XW performed the amniocentesis; KX, YY, and GC performed the DNA extraction and PCR. All authors have read and approved the final manuscript.

Funding

There was no funding for this study.

\section{Availability of data and materials}

We did not use new software, databases, or applications/tools in the manuscript, all results and figures have already provided in the manuscript.

\section{Ethics approval and consent to participate}

All research was approved by the institutional review board of Jiangxi maternal and child health hospital, Nanchang, China. Written informed consents for the use of medical information and genetic analysis were obtained from all the enrolled subjects.

\section{Consent for publication}

The enrolled adults provided signed consents for the permission of using their clinical information and sequencing data for research purposes and/or scientific publications. For the participants under 16 years old, written informed consent was obtained from their parents. For the participant died at adult stage (l:2), written informed consent was obtained from her daughters.

\section{Competing interests}

The authors declare that they have no competing interests.

\section{Author details}

${ }^{1}$ Prenatal Diagnosis Center, Jiangxi Provincial Maternal and Child Health Hospital, Nanchang 330006, Jiangxi, China. ${ }^{2}$ Central Laboratory, Jiangxi Provincial Maternal and Child Health Hospital, Nanchang 330006, Jiangxi, China.

Received: 17 September 2019 Accepted: 29 December 2019

Published online: 20 January 2020

\section{References}

1. Takaesu G, et al. TAB2, a novel adaptor protein, mediates activation of TAK1 MAPKKK by linking TAK1 to TRAF6 in the IL-1 signal transduction pathway. Mol Cell. 2000;5(4):649-58.

2. Thienpont $B$, et al. Haploinsufficiency of TAB2 causes congenital heart defects in humans. Am J Hum Genet. 2010;86(6):839-49.

3. Wade EM, et al. Mutations in MAP $3 K 7$ that Alter the activity of the TAK1 signaling complex cause Frontometaphyseal dysplasia. Am J Hum Genet. 2016;99(2):392-406.

4. Wade EM, et al. Autosomal dominant frontometaphyseal dysplasia: delineation of the clinical phenotype. Am J Med Genet A. 2017;173(7): 1739-46.

5. Richards S, et al. Standards and guidelines for the interpretation of sequence variants: a joint consensus recommendation of the American College of Medical Genetics and Genomics and the Association for Molecular Pathology. Genet Med. 2015;17(5):405-24.

6. Chen J, et al. A novel mutation of GATA4 (K300T) associated with familial atrial septal defect. Gene. 2016;575(2 Pt 2):473-7.

7. Adzhubei I, Jordan DM, Sunyaev SR. Predicting functional effect of human missense mutations using PolyPhen-2. Curr Protoc Hum Genet. 2013; Chapter 7:Unit7 20.

8. Kumar P, Henikoff S, Ng PC. Predicting the effects of coding nonsynonymous variants on protein function using the SIFT algorithm. Nat Protoc. 2009;4(7):1073-81.

9. Schwarz JM, et al. MutationTaster evaluates disease-causing potential of sequence alterations. Nat Methods. 2010;7(8):575-6.

10. Larkin MA, et al. Clustal W and Clustal X version 2.0. Bioinformatics. 2007; 23(21):2947-8

11. Weintraub RG, Semsarian C, Macdonald P. Dilated cardiomyopathy. Lancet. 2017:390(10092):400-14.

12. Bharucha $T$, et al. Sudden death in childhood cardiomyopathy: results from a long-term national population-based study. J Am Coll Cardiol. 2015;65(21): 2302-10.

13. Vasilescu C, et al. Genetic basis of severe childhood-onset cardiomyopathies. J Am Coll Cardiol. 2018;72(19):2324-38. 
14. Ritelli M, et al. A recognizable systemic connective tissue disorder with polyvalvular heart dystrophy and dysmorphism associated with TAB2 mutations. Clin Genet. 2018;93(1):126-33.

15. Salpietro $\mathrm{V}$, et al. A de novo $0.63 \mathrm{Mb} 6 \mathrm{q} 25.1$ deletion associated with growth failure, congenital heart defect, underdeveloped cerebellar vermis, abnormal cutaneous elasticity and joint laxity. Am J Med Genet A. 2015; 167A(9):2042-51.

16. Weiss $K$, et al. Familial TAB2 microdeletion and congenital heart defects including unusual valve dysplasia and tetralogy of fallot. Am J Med Genet A. 2015;167A(11):2702-6.

17. Cheng A, et al. 6q25.1 (TAB2) microdeletion syndrome: Congenital heart defects and cardiomyopathy. Am J Med Genet A. 2017;173(7):1848-57.

18. Ackerman JP, et al. Whole exome sequencing, familial genomic triangulation, and systems biology converge to identify a novel nonsense mutation in TAB2-encoded TGF-beta activated kinase 1 in a child with Polyvalvular syndrome. Congenit Heart Dis. 2016;1 1(5):452-61.

19. Caulfield $T R$, et al. Protein molecular modeling techniques investigating nove TAB2 variant R347X causing cardiomyopathy and congenital heart defects in multigenerational family. Mol Genet Genomic Med. 2018;6:666-72.

20. Goumans MJ, Ten Dijke P. TGF-beta Signaling in Control of Cardiovascular Function. Cold Spring Harb Perspect Biol. 2018;10(2). https://doi.org/10. 1101/cshperspect.a022210.

21. Boyer AS, et al. TGFbeta2 and TGFbeta3 have separate and sequential activities during epithelial-mesenchymal cell transformation in the embryonic heart. Dev Biol. 1999;208(2):530-45.

22. Azhar $\mathrm{M}$, et al. Ligand-specific function of transforming growth factor beta in epithelial-mesenchymal transition in heart development. Dev Dyn. 2009; 238(2):431-42

23. Geirsson A, et al. Modulation of transforming growth factor-beta signaling and extracellular matrix production in myxomatous mitral valves by angiotensin II receptor blockers. Circulation. 2012;126(11 Suppl 1):S189-97.

24. Disha $\mathrm{K}$, et al. Transforming growth factor Beta-2 mutations in Barlow's disease and aortic dilatation. Ann Thorac Surg. 2017;104(1):e19-21.

25. Hirata Y, et al. Post-Translational Modifications of the TAK1-TAB Complex. Int J Mol Sci. 2017;18(1). https://doi.org/10.3390/ijms18010205.

\section{Publisher's Note}

Springer Nature remains neutral with regard to jurisdictional claims in published maps and institutional affiliations.

Ready to submit your research? Choose BMC and benefit from:

- fast, convenient online submission

- thorough peer review by experienced researchers in your field

- rapid publication on acceptance

- support for research data, including large and complex data types

- gold Open Access which fosters wider collaboration and increased citations

- maximum visibility for your research: over $100 \mathrm{M}$ website views per year

At BMC, research is always in progress.

Learn more biomedcentral.com/submissions 